\title{
The RNA Pol II sub-complex hsRpb4/7 is required for viability of multiple human cell lines
}

\author{
Yang Zhao, Kim K.C. Li, King Pan Ng, Chi Ho Ng, Kevin A.W. Lee ${ }^{\bowtie}$ \\ Division of Life Science, The Hong Kong University of Science and Technology, Sai Kung, Hong Kong S.A.R. China \\ $\triangle$ Correspondence: bokaw@ust.hk \\ Received August 12, 2012 Accepted September 10, 2012
}

\begin{abstract}
The evolutionarily conserved RNA Polymerase II Rpb4/7 sub-complex has been thoroughly studied in yeast and impacts gene expression at multiple levels including transcription, mRNA processing and decay. In addition Rpb4/7 exerts differential effects on gene expression in yeast and Rpb4 is not obligatory for yeast (S. cerevisiae) survival. Specialised roles for human (hs) Rpb4/7 have not been extensively described and we have probed this question by depleting hsRpb4/7 in established human cell lines using RNA interference. We find that Rpb4/7 protein levels are inter-dependent and accordingly, the functional effects of depleting either protein are co-incident. hsRpb4/7 exhibits gene-specific effects and cells initially remain viable upon hsRpb4/7 depletion. However prolonged hsRpb4/7 depletion is cytotoxic in the range of cell lines tested. Protracted cell death occurs by an unknown mechanism and in some cases is accompanied by a pronounced elongated cell morphology. In conclusion we provide evidence for a gene-specific role of hsRpb4/7 in human cell viability.
\end{abstract}

KEYWORDS RNA Polymerase II, hsRpb4/7 sub-complex, gene expression, cell viability, cell shape

\section{INTRODUCTION}

The Rpb4 and Rpb7 subunits of RNA Polymerase II (Pol II) are of particular interest because, in contrast to other Pol II components, they form a dissociable sub-complex that has specialised gene-specific effects (Choder, 2004; Sampath and Sadhale, 2005). There is significant structural conservation of Rpb4/7 across the animal and plant kingdoms but current understanding of Rpb4/7 function has come mostly from yeast.

S. cerevisiae (sc) Rpb4/7 form a dissociable complex with Pol II (Edwards et al., 1991; Khazak et al., 1998) and scRpb4/7 activates promoter-specific transcription in vitro (Edwards et al., 1991; Jensen et al., 1998). The Rpb4/7 sub-complex also interacts with general transcription factors TFIIB and TFIIF (Bushnell and Kornberg, 2003; Chung et al., 2003), transcriptional activator proteins (Bertolotti et al., 1998; Petermann et al., 1998; Shen et al., 1999; Todorova, 2009) and the Pol II CTD phosphatase Fcp1 (Kimura et al., 2002; Kamenski et al., 2004). Intriguingly non-transcriptional functions of Rpb4/7 have also emerged (Choder, 2004; Sampath and Sadhale, 2005). Rpb4/7 binds to RNA as a heterodimer (Orlicky et al., 2001) and both proteins are intimately involved in coupling of transcription and RNA processing (Mitsuzawa et al., 2003; Runner et al., 2008). Second, Rpb4 (Kimura et al., 2001) and Rpb7 (Khazak et al., 1995; $\mathrm{Na}$ et al., 2003) can be found in the cytoplasm with Rpb4 mediating RNA export from the nucleus under stress conditions (Farago et al., 2003) and both Rpb4 and Rpb7 are involved in selective mRNA decay (Lotan et al., 2005; Goler-Baron et al., 2008). In terms of their biological roles, while scRpb4 and scRpb7 may not be identical (scRpb4 is conditional (Woychik and Young, 1989) and scRpb7 is essential (McKune et al., 1993)) both proteins are nonetheless involved in particular stress responses (Choder and Young, 1993; Sheffer et al., 1999; Maillet et al., 1999; Pillai et al., 2001) and are implicated in morphological changes that accompany nutrient starvation (Khazak et al., 1995; Pillai et al., 2003). Consistent with the conditional nature of scRpb4, global gene expression profiling in yeast indicates that a limited number of genes ( $2 \%$ of the genome) are affected by Rpb4 (Pillai et al., 2003), although other evidence points 
to a more global effect (Miyao et al., 2001).

In contrast to yeast, human (hs) Rpb4/7 function is not well characterised. Highly variable mRNA levels in several human tissues (Khazak et al., 1995; Schoen et al., 1997) may point to a non-essential and/or specialised function. hsRpb4/7 are also implicated in human cancer. The von Hippel-Lindau (VHL) tumor suppressor protein ( $\mathrm{Na}$ et al., 2003) directly interacts with and destabilises hsRpb7 via the ubiquitin-proteasome pathway. hsRpb7 also interacts physically (Bertolotti et al., 1998; Petermann et al., 1998; Todorova, 2009) and functionally (Zhou and Lee, 2001) with Ewing's family oncoproteins (Romeo and Dei Tos, 2010; Kovar, 2011) and may play a role in the Ewing's family of tumors. It is therefore of significance to understand the role of hsRpb4/7 in mammalian cell proliferation and survival.

Herein we describe experiments to assess the effect of depleting endogenous hsRpb4/7 in human tissue culture cells. Our results provide evidence for a concerted gene-specific role of hsRpb4 and hsRpb7 in survival of at least some human cells. The cytotoxic effect of hsRpb4/7 depletion precludes scrutiny of any specialised role for hsRpb4/7 in cell lines from Ewing's family tumors.

\section{RESULTS AND DISCUSSION}

\section{Identification of effective hsRpb4/7 siRNAs}

Multiple synthetic siRNAs targeting different hsRpb4 and hsRpb7 sequences were tested for silencing efficacy using a rapid and sensitive reporter assay employing Rpb4/7-EGFP targets and antibody to EGFP (Chau and Lee, 2007). For hsRpb7 four longer than normal 27mer siRNAs (R7L1-4) were tested because longer siRNAs are more potent (Kim et al., 2005) and thus can be used at concentrations (20 nmol/L) low enough to reduce potential off-target effects (Persengiev et al., 2004). Three of the above siRNAs (R7L1, R7L3 and $\mathrm{R} 7 \mathrm{~L} 4$ ) had strong silencing activity at $20 \mathrm{nmol} / \mathrm{L}$ (Fig. $1 \mathrm{~A}$ ) and one (R7L4) was very effective at $5 \mathrm{nmol} / \mathrm{L}$ (data not shown). Similarly for hsRpb4, three 27mer siRNAs (R4L1-3) were effective at $20 \mathrm{nmol} / \mathrm{L}$ (Fig. 1A) and one of these (R4L3) also worked well at $5 \mathrm{nmol} / \mathrm{L}$. R4L3 and R7L4 were used to establish conditions for depletion of endogenous hsRpb4 and hsRpb7. Both R4L3 and R7L4 (at $20 \mathrm{nmol} / \mathrm{L}$ ) were able to significantly deplete endogenous hsRpb4 and hsRpb7 (Fig. 1B) as detected using affinity purified rabbit polyclonal anti-Rpb7 and anti-Rpb4 antibodies.

\section{hsRpb7/4 expression is coordinated in the nucleus and cytoplasm}

Destabilisation of hsRpb7 protein by the tumor suppressor VHL protein (Na et al., 2003) suggests that post-transcriptional/ translational events may maintain low hsRpb7 protein levels in mammalian cells. Consistent with this suggestion our at- tempts to express an exogenous hsRpb7-EGFP fusion (Rpb7e, Fig. 2A) or essentially native hsRpb7 (modified only by a small KT3 epitope tag, Fig. 2B) resulted in poor expression. However co-expression with exogenous hsRpb4e (Fig. 2A) or KT3-tagged hsRpb4 (Fig. 2B) greatly elevated hsRpb7e (or hsRpb7) levels respectively and this effect was specific for hsRpb7 (Fig. 2A). Exogenous hsRpb4 did not noticeably increase endogenous hsRpb7 levels, presumably because exogenous hsRpb4 is expressed in only a fraction of cells and/or endogenous hsRpb7 is sequestered in relatively stable complexes with endogenous hsRpb4.

Similar relative levels of expression of hsRpb4 and hsRpb7 in different human tissues (Khazak et al., 1998) suggests that the endogenous genes/proteins might be coordinately expressed. To examine this possibility we observed protein levels following siRNA-mediated depletion. In the experiment shown, R4L3siRNA decreased Rpb4 and also Rpb7 levels (6.3-fold and 5.4-fold respectively) and
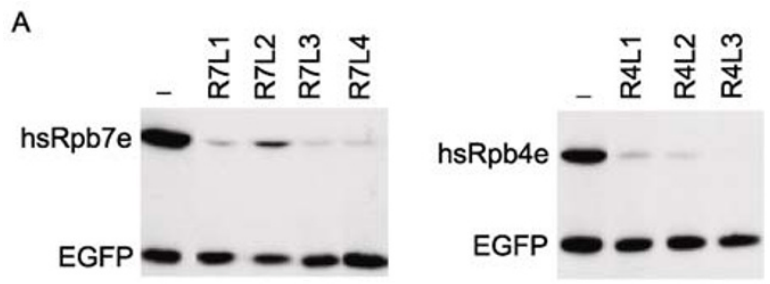

B

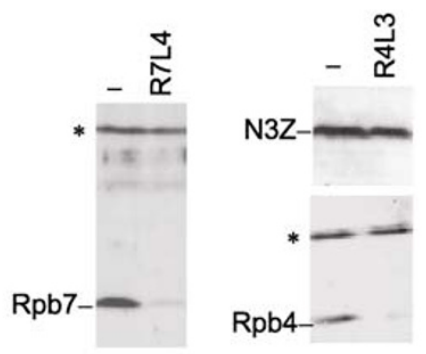

Figure 1. Potent siRNAs against hsRpb4/7 and depletion of the endogenous proteins. (A) HeLa cells in a 24-well tissue culture plate were co-transfected with equal amounts (300 $\mathrm{ng}$ each) of plasmid expression vectors for dsEGFP, Rpb4/7-EGFP fusions (hsRpb4/7e) and $20 \mathrm{nmol} / \mathrm{L}$ siRNAs (top) against hsRpb7 (R7L1-4) or hsRpb4 (R4L1-3). Silencing was assessed at $20 \mathrm{~h}$ post-transfection by Western blotting using EGFP antibody. (B) Depletion of endogenous hsRpb4 and hsRpb7. HeLa cells were transfected by calcium phosphate co-precipitation in the absence of siRNA (-) or with $20 \mathrm{nmol} / \mathrm{L}$ R7L4 (left) or R4L3 (right). Endogenous hsRpb4/7 were detected by Western blotting at $48 \mathrm{~h}$ (Rpb7) and $72 \mathrm{~h}$ (Rpb4) post-transfection, using hsRpb4 or hsRpb7 polyclonal antibodies (see materials and methods). A non-specific immunoreactive species $\left(^{*}\right)$ serves as a loading control. Cell viability was also assessed by expression of an exogenous protein (N3Z) via transfection following Rpb4-depletion. 
A

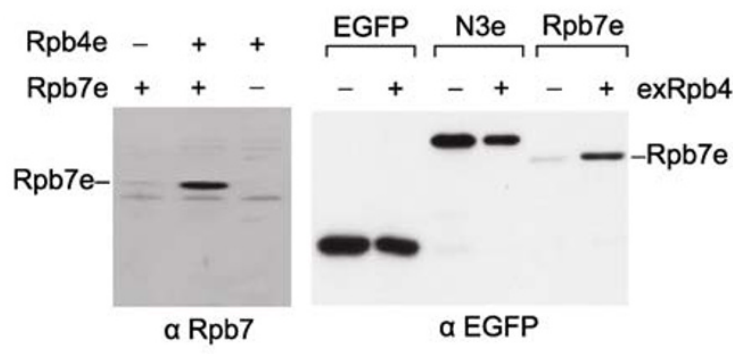

B

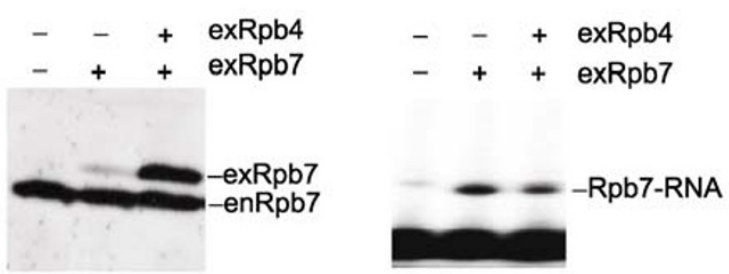

C

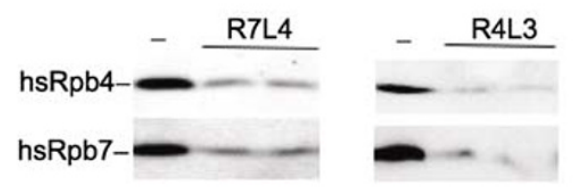

Figure 2. Effect of Rpb4 on Rpb7 levels. (A) Left hand side. Cells were transfected with expression vectors for hsRpb4e and hsRpb7e followed by Western blotting with anti-Rpb7. Right hand side: control experiment to test the effect of exogenous hsRpb4 (exRpb4) on proteins (EGFP and N3e) unrelated to hsRpb7. Proteins were detected using anti-EGFP. (B) HeLa cells were co-transfected with expression vectors for exogenous KT3-tagged hsRpb7 (exRpb7) or hsRpb7 plus hsRpb4 (exRpb4). The KT3 tag increases the size of exRpb7 sufficiently to allow distinction from endogenous hsRpb7 (enRpb7) on SDS gels. ex-Rpb7 and en-Rpb7 were detected using anti-hsRpb7 (left). Exogenous hsRpb7 mRNA (extracted from the same cells used for protein analysis) was detected by primer extension (right) using a ${ }^{32} \mathrm{P}$-labeled $\mathrm{S} 4$ primer (see materials and methods). (C) Depletion of endogenous hsRpb4 and hsRpb7 was achieved using R4L3 and R7L4 siRNAs (Fig. 1). hsRpb4/7 protein levels in duplicate protein samples were determined using hsRpb4 or hsRpb7 antibodies.

similarly R7L4siRNA decreased Rpb7 and also Rpb4 (3.7-fold and 3.6-fold respectively). Thus expression of endogenous hsRpb7 and hsRpb4 proteins is inter-dependent (Fig. 2C). In light of role of Rpb4 in mRNA export in yeast (Farago et al., 2003) we asked whether hsRpb4 increases hsRpb7 RNA levels. Exogenous hsRpb7 RNA transcript levels were monitored by primer extension but no effect of hsRpb4 protein was detected (Fig. 2B). Thus the stimulatory effect of hsRpb4 on hsRpb7 protein levels is exerted after mRNA formation and perhaps most likely via hsRpb7 stabilisation within the Rpb4/7 heterodimer. In this event hsRpb4 binding to hsRpb7 would be required for increasing Rpb7 and the assay described above using exogenous proteins will allow this question to be addressed.
Another significant aspect of Rpb4/7 expression concerns sub-cellular location. While some Rpb4/7 is necessarily nuclear for transcription in both yeast and human cells (Khazak et al., 1998), Rpb4 is also found in the cytoplasm in S. pombe (Kimura et al., 2001), shuttles between the nucleus and the cytoplasm in S. cerevisiae (Selitrennik et al., 2006) and is even predominantly cytoplasmic under some stress conditions (Farago et al., 2003). Previously, immuno-staining of endogenous hsRpb4/7 was not conclusive either due to cross-reacting antigens (for Rpb7) or lack of signal for Rpb4 (Khazak et al., 1998). We have also been unable to clearly detect endogenous hsRpb4/7 in mammalian cells using the antibodies described herein. We therefore employed an exogenous Rpb4-EGFP fusion (Rpb4e) and hsRpb7-red fluorescent protein (Rpb7r) to probe their sub-cellular location (Fig. 3). Consistent with the expression levels for the native proteins (Fig. 2), Rpb7r was only observed in cells expressing Rpb4e, thus confirming the stimulatory effect of hsRpb4 on hsRpb7. In addition to nuclear localisation, some cells showed striking cytoplasmic expression with apparent co-localisation of $\mathrm{Rbp} 4 \mathrm{e}$ and $\mathrm{Rpb7r}$ and an even distribution throughout the cytoplasm (Fig. 3). A small percentage of cells exhibited highly localised and granular cytoplasmic expression pattern near the nucleus and again with apparent co-localisation of Rbp4 and Rpb7 (Fig. 3). The latter phenomenon may reflect the situation in yeast whereby Rpb4/7 localise to cytoplasmic $P$ bodies (Lotan et al., 2005) that are the site of mRNA decay (Ingelfinger et al., 2002). Overall the subcellular pattern of

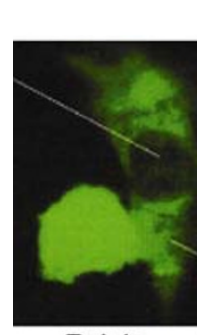

Rpb4e

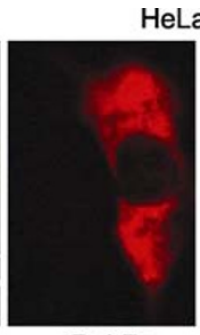

$\mathrm{Rpb} 7 \mathrm{r}$

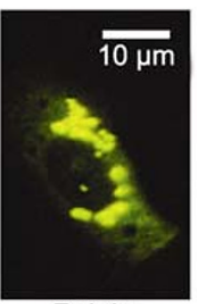

Rpb4e

\section{eLa cells}

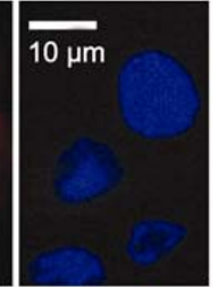

Hoechst

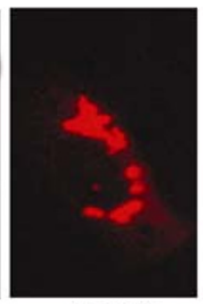

Rpb7r

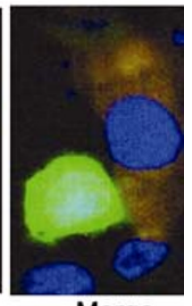

Merge
Figure 3. Subcellular location of tagged exogenous hsRpb4/7. HeLa cells were transfected with plasmids expressing an hsRpb4-EGFP fusion (Rpb4e) and hsRpb7 fused to a red fluorescent protein (Rpb7r). Images were collected at $36 \mathrm{~h}$ post-transfection. The upper four panels show the same field with cell nuclei stained by Hoechst. The two lower panels show the same cell from another field. A $10-\mu \mathrm{m}$ size bar is included. 
hsRpb4/7 in human cells appears to share common features with yeast and suggests that both nuclear and cytoplasmic functions have been evolutionarily conserved.

\section{Preliminary Gene Expression Profiling in Rpb7-depleted human cells}

Global gene expression profiling (GEP) in yeast (S. cerevisiae) indicates (at least under some conditions) that scRpb4 affects a limited number of genes corresponding to $\sim 2 \%$ of the genome (Pillai et al., 2003). To evaluate the global effect of hsRpb4/7 in human cells we performed GEP in Rpb7-depleted HeLa cells using Agilent Human Whole Genome oligo arrays (see materials and methods). Validity of the GEP was verified by scoring of a $77 \%$ reduction in hsRpb7RNA in the GEP analysis and determination of a similar reduction $(90 \%)$ in hsRpb7 protein in depleted versus control cells (Fig. 1A). In Rpb7-depleted HeLa cells GEP resulted in scoring of 429 up-regulated signals or only $\sim 1 \%$ of the genome (using a 2-fold threshold) and only 88 up-regulated genes (3-fold threshold). The limited number of genes affected cannot be attributed to partial Rpb7-depletion because expression of eighteen genes was increased between 6 -fold and 34-fold. Identification of up-regulated genes is consistent with the role of Rpb4/7 in selective mRNA decay in yeast (Lotan et al., 2005; Goler-Baron et al., 2008). Similarly Rpb7-depletion identified 261 down-regulated signals (2-fold threshold) and only 71 down-regulated genes (2.5-fold threshold). Significant impact on expression of a limited portion of cellular genes further indicates that hsRpb4/7 has a gene-specific rather than general role in human cells.

Because depletion of hsRpb4/7 ultimately leads to cell death (see below) it is not a simple task to identify key primary target genes for hsRpb4/7. Nonetheless mapping differentially expressed genes to the Gene Ontology (GO) database (genes significantly enriched in $\mathrm{GO}$ categories, t-test $p<0.05$ ) resulted in selection of several GO categories and some general features may be insightful. Firstly considering the cytotoxic effect of hsRpb7 depletion (see below) it may be significant that several genes implicated in cell cycle arrest/non-apoptotic cell death (CDKN1A, SESN1, HEXIM1) are significantly up-regulated (3-4 folds). Secondly a significant proportion $(15 \%)$ of genes involved in various biosynthetic/metabolic pathways are altered by hsRpb7-depletion and this may mirror the general defect in expression of metabolic genes in yeast in the absence of Rpb4 (Pillai et al., 2003).

\section{Effect of hsRpb4/7 depletion on human cell viability}

Variable mRNA hsRpb4/7 RNA levels in several human tissues (Khazak et al., 1995; Schoen et al., 1997) suggests that hsRpb7 might not be essential for cell survival. As a prerequisite for examining the above question we confirmed expression of hsRpb4 and hsRpb7 proteins in several human cell lines (Jeg3, MST1, Kao, DTC1, GG62) in addition to HeLa (Fig. 4). Both proteins were expressed at reasonably similar levels in nuclear extracts from all cells tested ( 3-fold variation, normalised for cell number) and to date we have not identified any cell lines lacking hsRbp4/7. In addition the relative expression of hsRpb4/hsRpb7 is broadly similar comparing different cells (relative signals in Fig. 4 reflect the different antibodies and not the absolute hsRpb4/7 ratio). It thus appears from our preliminary analysis that smaller variations in hsRpb4/7 protein levels in different cell lines may not reflect the more significant differences in mRNA levels observed in human tissues (Khazak et al., 1995; Schoen et al., 1997) or cell lines (K.A.W.L, unpublished results).

Initially we did not observe any cytotoxicity in Hela, Jeg3, DTC1 or GG62 cells following short-term hsRpb4/7 depletion (up to $72 \mathrm{hr}$ ) using synthetic siRNAs. This prompted us to examine the effect of prolonged depletion via stable introduction of constitutive, selectable short-hairpin (sh) RNAs targeting hsRpb4/7 (Fig. 4). The gene silencing efficacy of different shRNAs against hsRpb4 (R4sh, corresponding to R4L3 siRNA), hsRpb7 (R7sh, corresponding to R7L4 siRNA) and a negative control shRNA (nc) was again established using a transient assay in HeLa cells and scoring depletion of hsRpb7e and hsRpb4e reporter proteins (Fig. 4). For cell viability assays, stable cell transformants were selected by G418 and viable colonies stained with Coomassie Blue (12 days post selection). We first tested two cell lines (Kao and MST1) derived from Soft Tissue Clear Cell Sarcoma (ST-CCS), a member of the Ewing's family of tumors (Zucman et al., 1993; Dim et al., 2007; Wang et al., 2009). Both hsRpb4 and hsRpb7 shRNAs were toxic to Kao (data not shown) and MST1 cells (Fig. 4). However the same result was obtained for two non-ST-CCS cell lines, HeLa and Jeg3 (Fig. 4). The few viable colonies that survive following introduction of shR4/7 presumably arise due to low frequency loss/inactivation of the short-hairpin part of the transgene while retaining neomycin resistance. Together with other observations (see below and Fig. 6) this indicates that the ultimate outcome of prolonged Rpb4/7 depletion is cell death.

To provide rigorous evidence that the cytotoxic effect observed is due to hsRpb7 depletion, we tested a panel of hsRpb7 shRNAs (m2, m6, $\mathrm{m} 7$ and $\mathrm{m} 8$ ) with discrete mutations in the 5 region of the siRNA guide strand (Fig. 5). Mutants $\mathrm{m} 6$ and $\mathrm{m} 7$ retain both Rpb7 silencing and cytotoxicity while mutants $\mathrm{m} 2$ and $\mathrm{m} 8$ lose both functions (Fig. 5). Thus for several hsRpb7 shRNAs tested there is a correlation between silencing activity and cytotoxicity, indicating that the cytotoxic effect is due to lack of hsRpb4/7. In summary these findings indicate that $\mathrm{hsRpb} 4 / 7$ is required for survival of several human cell lines.

\section{Morphological changes induced by hsRpb4/7 depletion}

The eventual outcome of hsRpb4/7 depletion is cell death but 


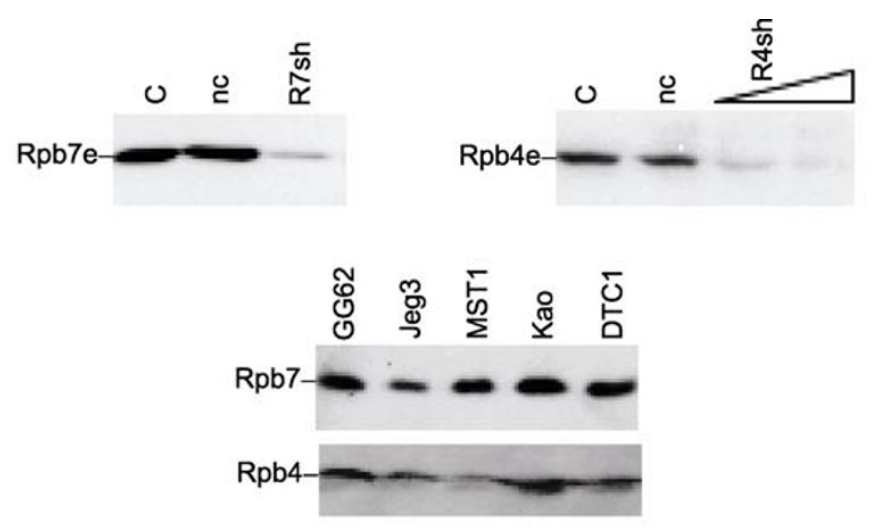

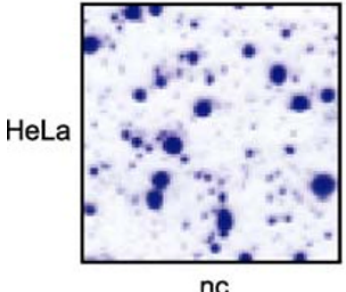

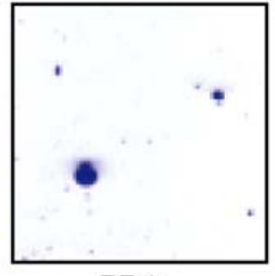

R7sh

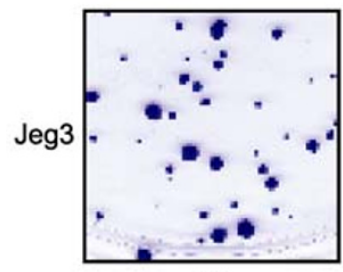

nc

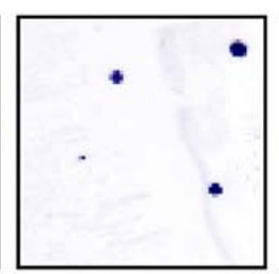

R7sh

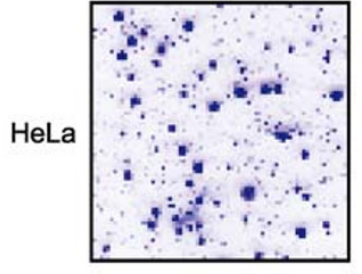

nc

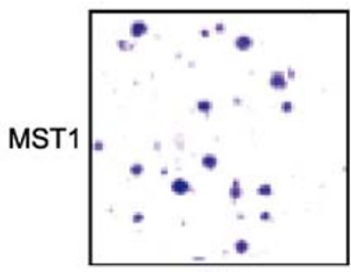

nc

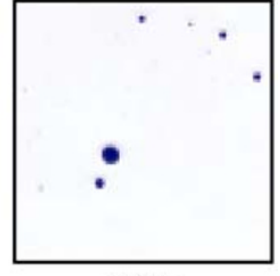

R4sh

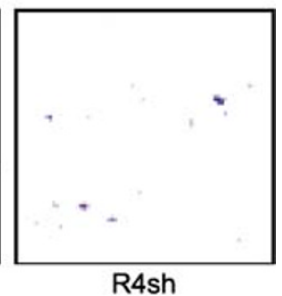

Figure 4. Effect of hsRpb4 and hsRpb7 on cell viability. Top. HeLa cells were co-transfected with expression vectors for hsRpb7e or hsRpb4e and shRNAs against hsRpb7 (R7sh) or hsRpb4 (shR4) or a negative control shRNA (nc). Rpb7e and Rpb4e depletion in normalised protein extracts was scored by Western blot using EGFP antibody. Middle. Endogenous hsRpb7 and hsRpb4 in nuclear extracts from the indicated cells were detected by Western blot. Lower panels. Stable cell transformants from HeLa, Jeg3 or MST1 cells (derived from the Ewing's family Soft Tissue Clear Cell Sarcoma). Cells were transfected with vectors for the shRNAs indicated or a negative control shRNA (nc). G418 resistant colonies were stained with Coomassie Blue R250 at 12 days post-transfection.

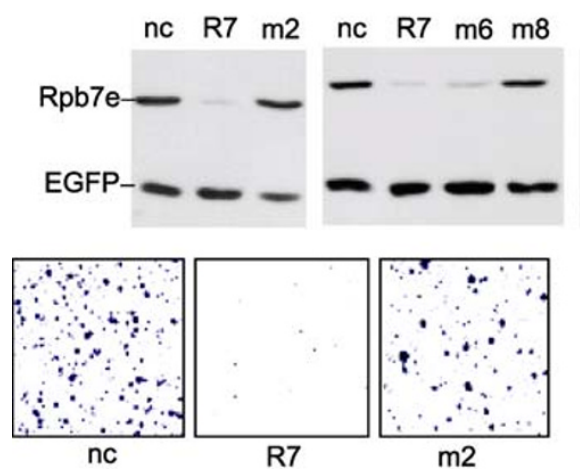

R7
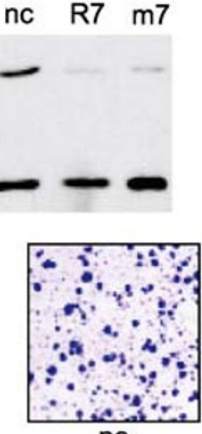

nc

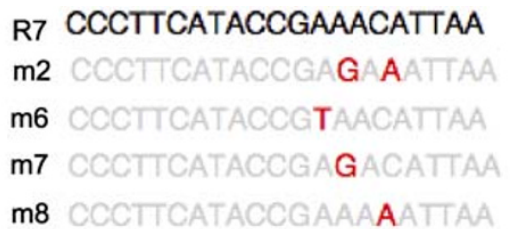

m8 CCCTTCATACCGAAAAATTAA

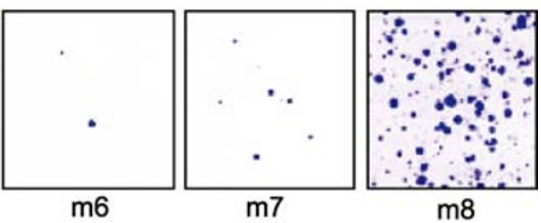

Figure 5. Specificity of R7sh effect on cell viability. Different R7sh mutants ( $\mathrm{m} 2, \mathrm{~m} 6, \mathrm{~m} 7$ and $\mathrm{m} 8$ ) were tested for gene silencing using an hsRpb7-EGFP reporter (top) and effect on cell viability (bottom). For conditions see Fig. 4. Silencing assays included an expression plasmid for EGFP as a control for transfection efficiency and sample loading. 
our results provide little insight into the mechanism involved. In addition the apparent lack of an acute cytotoxic response to hsRpb4/7-depletion may point to an indirect effect. It is of interest however that in some cells (HeLa) we observed a pronounced elongated cell morphology for both hsRpb4 and hsRpb7-depleted cells that persists for a substantial period (several days) prior to cell death (Fig. 6). Western blot analysis confirmed that hsRpb7 protein levels remain depleted ( 6-fold, normalised for cell number) in the above cells prior to cell death (Fig. 6). Because the cells studied normally grow in clusters with close cell-cell contact (even at low density, for example under G418 selection, Fig. 6) it cannot be ruled out that the striking morphology of hsRpb4/7-depleted cells results indirectly from an alteration of cell adhesion properties. The few viable colonies that survive following introduction of shR4/7 look like normal HeLa cells and grow in clusters (i.e. they are completely different compared with the elongated shR4/7 transformants that slowly die). These residual colonies therefore represent background and presumably arise due to low frequency loss/inactivation of the short-hairpin part of the transgene. Thus we suggest that prolonged depletion of Rpb4/7 ultimately leads to cell death.

In relation to the altered morphology of Rpb4/7-depleted HeLa cells it is intriguing that hsRpb7 was originally identified for the ability to enhance pseudohyphal growth in yeast (S. cerevisiae) under nitrogen starvation (Khazak et al., 1995). Such morphological changes are postulated to protect cells by enhancing the probability of finding a nutrient source with pseudohyphal cells being bigger and markedly elongated (Gimeno et al., 1992). S. cerevisiae cells lacking Rpb4 are also more prone to generate pseudohyphae (Pillai et al., 2003) and the fission yeast S. pombe exhibit elon- gated features and a deficiency in cell separation when Rpb4 expression is low (Sharma et al., 2006). It will be interesting to further characterise the hsRpb4/7-dependent morphological effects that we have described (Fig. 6) and to see if they reflect an evolutionarily conserved response mechanism.

\section{CONCLUSION}

We have directly addressed the function of $\mathrm{Rpb} 4 / 7$ in human cells and our results indicate that hsRpb4/7 have gene-specific effects but are also more generally required for human cell survival (at least in cultured cell lines). The latter finding so far precludes examination of a more specialised role of hsRpb4/7 in Ewing's family tumors. Inter-dependence of hsRpb4 and hsRpb7 protein levels and coincident sub-cellular localisation points to the concerted action of hsRpb4 and hsRpb7 via heterodimeric hsRbp4/7. It is likely that stabilisation of hsRpb7 requires interaction with hsRpb4 and the assay described herein, using exogenous hsRpb4/7, will allow the mechanism to be elucidated.

Our results demonstrate that several properties of hsRpb4/7 broadly mirror the well established characteristics of Rpb4/7 in yeast and thus that both nuclear and cytoplasmic Rpb4/7 functions have been evolutionarily conserved. In addition, the pronounced elongated cell morphology observed in hsRpb4 and hsRpb7-depleted cells prior to death may resemble the enhancement of pseudohyphal growth in yeast observed under nitrogen starvation. It will be interest to further characterise the Rpb4/7-dependent morphological effects in mammalian cells to determine whether they reflect an evolutionarily conserved response mechanism.

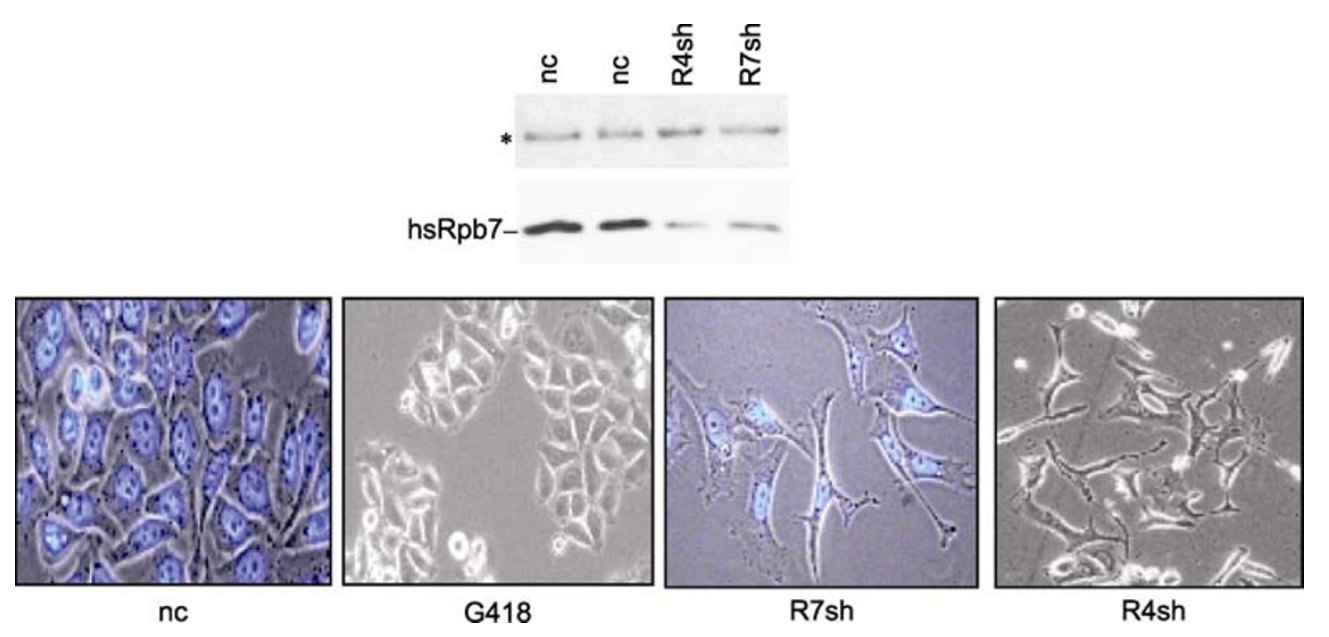

Figure 6. Morphology of hsRpb4/7-depleted HeLa cells. Top. hsRpb7 levels in normalized amounts of HeLa cells transfected with control shRNA vector (nc), shRpb7 (R7sh) or shRpb4 (R4sh) and selected with G418. A non-specific immunoreactive species $\left({ }^{*}\right)$ serves as a loading control. Bottom. Bright field images of HeLa cells after 6 days of G418 selection. Cells were transfected with control shRNA vector (nc), R7sh or R4sh. DAPI staining of nuclei in nc cells and Rpb7-depleted cells is also shown. Cells treated with G418 at lower cell density (G418) are also shown to aid comparison with the effect of R4/7sh. 


\section{MATERIALS AND METHODS}

\section{Plasmids}

Protein expression vectors were derived from pSG424 (Sadowski and Ptashne, 1989) and proteins tagged with the epitope for monoclonal antibody KT3 (MacArthur and Walter, 1984) or from pd2EGFP-N1 expressing destabilised EGFP (Clontech). EGFPtagged phsR7T and phsR4T express KT3-tagged hsRpb7 and hsRpb4 respectively. $\mathrm{pR} 7 \mathrm{E}$ and $\mathrm{pR} 4 \mathrm{E}$ express EGFP-tagged hsRpb7/hsRpb4. pN3e expresses the N-terminal 167 residues of the EAD tagged with EGFP. All plasmids expressing shRNAs were constructed by inserting synthetic gel-purified DNA oligonucleotides containing the shRNA sequence between the BamH1 and Hind3 sites of pSilencer 2.1-U6neo (Life Technologies).

\section{Cell lines and cell culture}

All cells were maintained in DME and $10 \%$ fetal bovine serum. ST-CCS cell lines MST1 and Kao are previously described (Li et al., 2003). For selection of stable transformants DNA was introduced by calcium phosphate/DNA co-precipitation, using $10 \mu \mathrm{g}$ of pSilencer 2.1-U6neo plasmid as positive control (or test shRNA plasmid) and $10 \mu \mathrm{g}$ of $\mathrm{pGem} 3$ as carrier. At $48 \mathrm{~h}$ post-transfection $500 \mu \mathrm{g} / \mathrm{mL}$ G418 was added the culture medium followed by a change of media and fresh drug every two days. Stable colonies were fixed and stained with Coomassie Blue R250.

\section{SiRNAs}

Synthetic siRNAs contained single strands of $27 \mathrm{nt}$ and $29 \mathrm{nt}$ producing double stranded siRNAs with a 2-nucleotide (UU) 3' overhang. The sequence for the most effective hsRpb7siRNA (R7L4) is 5'-UGCACAGGGAAGUAUGGCUUUGUAAUUUU-3' (passenger strand) and 5'-AAUUACAAAGCCAUACUUCCCUGUGCA-3' (guide strand). The sequence for the most effective hsRpb4siRNA (R4L3) is 5'-UACACAGCCCGUUUCAGUCGUUUCAAAUU-3' (passenger strand) and 5'-UUUGAAACGACUGAAACGGGCUGUGUA-3' (guide strand). shRNAs expressed from pSilencer 2.1-U6neo were designed to produce in vivo processed siRNAs with guide strands close to or identical with siRNAs R7L4 and R4L3. siRNAs were evaluated in HeLa cells using an EGFP-fusion reporter assay (Chau and Lee, 2007). Briefly cells in 24 well tissue culture plates were co-transfected with plasmids expressing the test protein fused to destabilised-EGFP and pd2EGFP-N1 (300 ng each) and synthetic siRNAs using Lipofectamine 2000 (Life Technologies). At 20 h post-transfection cells were harvested by trypsinisation, washed with PBS and then lysed in buffer containing $20 \mathrm{mmol} / \mathrm{L}$ Tris- $\mathrm{Cl}, \mathrm{pH} 7.5 ; 50 \mathrm{mmol} / \mathrm{L} \mathrm{NaCl} ; 1$ $\mathrm{mmol} / \mathrm{L}$ EDTA, $\mathrm{pH}$ 8; $1 \mathrm{mmol} / \mathrm{L}$ EGTA, $\mathrm{pH} 7 ; 1 \%$ Triton X-100 and complete protease inhibitor cocktail (Roche). Lysed cells were centrifuged at $13,000 \mathrm{~g}$ for $10 \mathrm{~min}$ and the cell lysate immediately adjusted to $1 \times$ SDS gel loading buffer for Western blot analysis.

\section{Western blotting and primer extensions}

Western blotting was performed in PBS containing $3 \%$ dried milk.
Endogenous hsRpb4 and hsRpb7 were detected using affinity purified rabbit polyclonal antibodies raised against bacterially expressed hsRpb4 and hsRpb7. Exogenous tagged proteins were detected using primary mouse monoclonal antibody KT3 (MacArthur and Walter, 1984) or EGFP antibody (mouse monoclonal JL8, Clontech). Secondary antibodies were anti-mouse or anti-rabbit HRP conjugated (Amersham NA931) and blots were developed using an ECL kit (Amersham NA931). Primer extension of hsRpb7 mRNA was performed as previously described (Lee and Green, 1987) using a 20nt ${ }^{32}$ P-labeled primer (5'-CAGGATTTCGTGCTCTAGGG-3') complementary to a sequence near the $5^{\prime}$ end (position 127-146) of hsRpb7mRNA.

\section{Gene Expression Profiling (GEP)}

GEP analysis was performed by Welgene Biotech Ltd. (Taiwan) employing Agilent Human Whole Genome Oligo $4 \times 44 \mathrm{~K}$ arrays. Data were normalized by Rank-invariant LOWESS normalization using Feature Extraction software (Agilent Technologies) with subsequent data analysis using GeneSpring 7.3.1 (Agilent Technologies). For hsRpb7-depleted versus control samples, Rpb7 protein depletion was verified by Western blotting (Fig. 1) and RNA samples were prepared at $72 \mathrm{~h}$ post-transfection. For GEP analysis, 429 features (1.05\% of total) with "High Confidence Significant UP" (>2-fold, $P<$ 0.01 , rProcessed Signal $>100$ ) and 261 features with "High Confidence Significant Down" $(<0.5, P<0.01$, gProcessed Signal $>100)$ were selected.

\section{ACKOWLEDGEMENTS}

We greatly appreciate the gene profiling service provided Welgene Biotech Ltd. (Taiwan). This work was funded by Hong Kong Government Research Grants Council (Project No: HKUST 6412/06M) to K.A.W. Lee.

\section{REFERENCES}

Bertolotti, A., Melot, T., Acker, J., Vigneron, M., Delattre, O., and Tora, L. (1998). EWS, but not EWS-FLI-1, is associated with both TFIID and RNA polymerase II: interactions between two members of the tet family, EWS and hTAF(II)68, and subunits of TFIID and RNA polymerase II complexes. Mol Cell Biol, 18, 1489-1497.

Bushnell, D.A., and Kornberg, R.D. (2003). Complete 12-subunit RNA polymerase II at 4.1-A resolution: implications for the initiation of transcription. Proc Natl Acad Sci U S A 100, 6969-6973.

Chau, B. L. and Lee, K.A.W. (2007). Function and anatomy of plant siRNA pools derived from hairpin transgenes. Plant Methods, 3,13 .

Choder, M., and Young, R.A. (1993). A portion of RNA polymerase II molecules has a component essential for stress responses and stress survival. Mol Cell Biol 13, 6984-6991.

Choder, M. (2004). Rpb4 and Rpb7: subunits of RNA polymerase II and beyond. Trends in Biochem Sci 29, 674-681.

Chung, W.H., Craighead, J.L., Chang, W.H., Ezeokonkwo, C., Bareket-Samish, A., Kornberg, R.D., and Asturias, F.J. (2003). RNA polymerase II/TFIIF structure and conserved organization of the 
the initiation complex. Mol Cell 12, 1003-1013.

Dim, D.C., Cooley, L.D., and Miranda, R.N. (2007). Clear cell sarcoma of tendons and Aponeuroses: a review. Arch Pathol Lab Med 131, 152-156.

Edwards, A.M., Kane, C.M., Young, R.A., and Kornberg, R.D. (1991). Two dissociable subunits of yeast RNA Polymerase II stimulate the initiation of transcription at a promoter in vitro. $\mathrm{J}$ Biol Chem 266, 71-75.

Farago, M., Nahari, T., Hammel, C., Cole, C.N., and Choder, M. (2003). Rpb4p, a subunit of RNA polymerase II, mediates mRNA export during stress. Mol Biol Cell 14, 2744-2755.

Gimeno, C.J., Jungdahl, P.O., Styles, C.A., and Fink, G.R. (1992). Unipolar cell divisions in the yeast $S$. cerevisiae lead to filamentous growth, regulation by starvation an RAS. Cell 68, 1077-1090.

Goler-Baron, V., Selitrennik, M., Barkai, O., Haimovich, G., Lotan, R., and Choder, M. (2008). Transcription in the nucleus and mRNA decay in the cytoplasm are coupled processes. Genes Dev 22, 2022-2027.

Ingelfinger, D., Arndt-Jovin, D.J., Luhrmann, R., and Achsel, T. (2002). The human LSm1-7 proteins colocalize with the mRNA-degrading enzymes Dcp1/2 and Xrn1 in distinct cytoplasmic foci. RNA 8, 1489-1501.

Jensen, G.J., Meredith, G., Bushnell, D.A., and Kornberg, R.D. (1998) Structure of wild-type yeast RNA polymerase II and location of Rpb4 and Rpb7. EMBO J 17, 2353-2358.

Kamenski, T., Heilmeier, S., Meinhart, A., and Cramer, P. (2004). Structure and mechanism of RNA polymerase II CTD phosphatases. Mol Cell 15, 399-407.

Khazak, V., Sadhale, P.P., Woychik, N.A., Brent, R., and Golemis, E.A. (1995). Human RNA polymerase II subunit hsRPB7 functions in yeast and influences stress survival and cell morphology. Mol Biol Cell 6, 759-775.

Khazak, V., Estojak, J., Cho, H., Majors, J., Sonoda, G., Testa, J.R., and Golemis, E.A. (1998). Analysis of the interaction of the novel RNA Polymerase II (pol II) subunit hsRPB4 with its partner hsRPB7 and with pol II. Mol Cell Biol 18, 1935-1945.

Kim, D.H., Behlke, M.A., Rose, S.D., Chang, M.S., Choi, S., and Rossi, J.J. (2005). Synthetic dsRNA Dicer substrates enhance RNAi potency and efficacy. Nat Biotech 23, 222-226.

Kimura, M., Sakurai, H., and Ishihama, A. (2001). Intracellular contents and assembly states of all 12 subunits of the RNA polymerase II in the fission yeast Schizosaccharomyces pombe. Eur J Biochem 268, 612-619.

Kimura, M., Suzuki, H., and Ishihama, A. (2002). Formation of a carboxy-terminal domain phosphatase (Fcpl)/TFIIF/RNA polymerase II (pol11) complex in Schizosaccharomyces pombe involves direct interaction between Fcpl and the Rpb4 subunit of pol II. Mol Cell Biol 22, 1577-1578.

Kovar, H. (2011). Dr. Jekyll and Mr. Hyde: The two faces of the FUS/EWS/TAF15 protein family. Sarcoma, 837474.

Lee, K.A.W., and Green, M.R. (1987). A cellular transcription factor E4F1 interacts with an Ela-inducible enhancer and mediates constitutive enhancer function in vitro. EMBO J 22, 4249-4259.

Li, K.K.C., Goodall, J., Goding, C.R., Liao, S.K., Wang, C.H., Lin Y.C., Hiraga, H., Nojima, T., Nagashima, K, Schaefer, K.L, et al. (2003). The melanocyte inducing factor mitf is stably expressed in cell lines: from human clear cell sarcoma. British $\mathrm{J}$ Cancer 89,
1072-1078.

Lotan, R., Goler Baron, V. Harel-Sharvit, L., Duek, L., Melamed, D., and Choder, M. (2005). The RNA polymerase II subunit Rpb4p mediates decay of a specific class of mRNAs. Genes Dev 19, 3004-3016.

MacArthur, H., and Walter, G. (1984). Monoclonal antibodies specific for the carboxy terminus of Simian virus 40 large T antigen. J Virol 52, 483-491.

Maillet, I., Buhler, J.M., Sentenac, A., and Labarre, J. (1999). Rpb4p is necessary forRNA polymerase II activity at high temperature. J Biol Chem 274, 22586-22590.

McKune, K., Richards, K.L., Edwards, A.M., Young, R.A., and Woychik, N.A. (1993). RPB7, one of two dissociable subunits of yeast RNA polymerase II, is essential for cell viability. Yeast 9, 295-299.

Mitsuzawa, H., Kanda, E., and Ishihama, A. (2003). Rpb7 subunit of RNA polymerase II interacts with an RNA-binding protein involved in processing of transcripts. Nucleic Acids Res 31, 4696-4701.

Miyao, T., Barnett, J.D., and Woychik, N.A. (2001). Deletion of the RNA polymerase subunit RPB4 acts as a global, not stress-specific, shut-off switch for RNA polymerase II transcription at high temperatures. J Biol Chem 276, 46408-46413.

$\mathrm{Na}$, X., Duan, H.O., Messing, E.M., Schoen, S.R., Ryan, C.K., di Sant'Agnese PA, Golemis EA, Wu G. (2003). Identification of the RNA polymerase II subunit hsRPB7 as a novel target of the von Hippel-Lindau protein. EMBO J 22, 4249-4259.

Orlicky, S.M., Tran, P.T., Sayre, M.H., and Edwards, A.M. (2001). Dissociable Rpb4-Rpb7 subassembly of RNA polymerase II binds to single-strand nucleic acid and mediates a post-recruitment step in transcription initiation. J Biol Chem 276, 10097-10102.

Persengiev, S.P., Zhu, X., and Green, M.R. (2004). Nonspecific, concentration-dependent stimulation and repression of mammalian gene expression by small interfering RNAs (siRNAs). RNA 10, 12-18.

Petermann, R., Mossier, B.M., Aryee, D.N.T., Khazak, V., Golemis, E.A., and Kovar, H. (1998). Oncogenic EWS-Fli1 interacts with hsRPB7, a subunit of human RNA polymerase II. Oncogene 17, 603-610.

Pillai, B., Sampath, V., Sharma, N., and Sadhale, P. (2001). Rpb4, a non-essential subunit of core RNA polymerase II of Saccharomyces cerevisiae is important for activated transcription of a subset of genes. J Biol Chem 276, 30641-30647.

Pillai, B., Verma, J., Abraham, A., Francis, P., Kumar, Y., Tatu, U. Brahmachari, S.K., and Sadhale, P. (2003). Whole genome expression profiles of yeast RNA polymerase II core subunit, Rpb4, in stress and non-stress conditions. J Biol Chem 278, 3339-3346.

Romeo, S., and Dei Tos, A.P. (2010). Soft tissue tumors associated with EWSR1 translocation. Virchows Arch 456, 219-234.

Runner, V.M., Podolny, V., and Buratowski, S. (2008). The Rpb4 subunit of RNA polymerase II contributes to cotranscriptional recruitment of 3' processing factors. Mol Cell Biol 28, 1883-1891.

Sadowski, I., and Ptashne, M. (1989). A vector for expressing GAL4 (1-147) fusions in mammalian cells. Nucleic Acids Res 17, 7539.

Sampath, V., and Sadhale, P. (2005). Rpb4 and Rpb7: a sub-complex integral to multi-subunit RNA polymerases performs a multitude of functions. IUBMB Life 57, 93-102,

Schoen, T.J., Chandrasekharappa, S.C., Guru, S.C., Mazuruk, K., Chader, G.J., and Rodriguez, I.R. (1997). Human gene for the 
RNA polymerase II seventh subunit (hsRPB7): structure, expression and chromosomal localization. Biochim Biophys Acta 1353, 39-49.

Selitrennik, M., Duek, L., Lotan, R., and Choder, M. (2006). Nucleocytoplasmic shuttling of the Rpb4p and Rpb7p subunits of Saccharomyces cerevisiae RNA polymerase II by two pathways. Eucaryot Cell 5, 2092-2103.

Sharma, N., Marguerat, S., Mehta, S., Watt, S., and Bahler, J. (2006). The fission yeast Rbp4 subunit of RNA Polymerase II plays a specialised role in cell separation. Mol Gen Genomics 276, 545-554.

Sheffer, A., Varon, M., and Choder, M. (1999). Rpb7 can interact with RNA polymerase II and support transcription during some stresses independently of Rpb4. Mol Cell Biol 19, 2672-2680.

Shen, X., Bubulya, A., Zhou, X., Khazak, V., Golemis, E.A., and Shemshedini, L. (1999). Ligand-Free RAR can interact with the RNA polymerase II subunit hsRPB7 and repress transcription. Endocrine 10, 281-289.
Todorova, R. (2009). In vitro interaction between the N-terminus of the Ewing's sarcoma protein and the subunit of RNA polymerase II hsRPB7. Mol Biol Rep 36, 1269-1274.

Wang, W., Mayordomo, E., Zhang, W., Hernandez, V.S., Tuvin, D., Garcia, L. Lev, D.C., Lazar, A.J.F., and Lopez-Terrada, D. (2009). Detection and characterization of EWSR1/ATF1 and EWSR1/CREB1 chimeric transcripts in clear cell sarcoma (melanoma of soft parts). Mod Pathol 22, 1201-1209.

Woychik, N.A., and Young, R.A. (1989). RNA polymerase II subunit RPB4 is essential for high-and low-temperature yeast cell growth. Mol Cell Biol 9, 2854-2859.

Zhou, H., and Lee, K.A.W. (2001). An hsRPB4/7-dependent yeast assay for trans-activation by the EWS. Oncogene 20, 1519-1524.

Zucman, J., Delattre, O., Desmaze, C., Epstein, A.L., Stenman, G., Speleman, F., Fletchers C.D., Aurias, A., and Thomas, G. (1993). EWS and ATF-1 gene fusion induced by $t(12 ; 22)$ translocation in malignant melanoma of soft parts. Nat Genet 4, 341-345. 